Meta

Journal des traducteurs

Translators' Journal

\title{
Science, dénomination et partage du pouvoir : le cas des éponymes
}

\section{Uzoma Chukwu}

Volume 41, numéro 4, décembre 1996

La dénomination

URI : https://id.erudit.org/iderudit/002242ar

DOI : https://doi.org/10.7202/002242ar

Aller au sommaire du numéro

Éditeur(s)

Les Presses de l'Université de Montréal

ISSN

0026-0452 (imprimé)

1492-1421 (numérique)

Découvrir la revue

Citer cet article

Chukwu, U. (1996). Science, dénomination et partage du pouvoir : le cas des éponymes. Meta, 41(4), 590-603. https://doi.org/10.7202/002242ar
Résumé de l'article

Si le recours à l'éponyme est un procédé de formation de termes très productif dans certains domaines, il reste néanmoins un phénomène très peu étudié par les linguistes et, plus particulièrement, les terminologues qui semblent renvoyer la balle à la logique. D'autre part, à en juger par les invectives fréquentes contre les éponymes, ils n'ont, dans l'ensemble, pas bonne presse chez les utilisateurs de la langue. Cependant, envisagée par rapport à des domaines particuliers, la situation paraît beaucoup plus nuancée. En médecine, par exemple, ils rencontrent beaucoup d'hostilité parce qu'ils sont perçus comme des facteurs perturbateurs de la terminologie du domaine, pour l'essentiel transparente puisque largement fondée sur la composition savante. En histoire naturelle, ils sont plutôt bien intégrés et forment même l'ossature du système binominal de Linné, alors qu'en physique (et en mathématiques), ils ne semblent pas susciter de sentiment particulier. Mais si le procédé se maintient dans ce climat a priori défavorable, c'est d'abord parce qu'il étend considérablement la capacité de dénomination des langues naturelles en mettant à leur disposition tout le répertoire des patronymes en nombre quasi illimité. Son maintien s'explique aussi par le fait qu'il participe au processus de régulation interne de la science en permettant à celle-ci de reconnaître les mérites des siens.
Ce document est protégé par la loi sur le droit d'auteur. L'utilisation des services d’Érudit (y compris la reproduction) est assujettie à sa politique d'utilisation que vous pouvez consulter en ligne.

https://apropos.erudit.org/fr/usagers/politique-dutilisation/ 


\title{
SCIENCE, DÉNOMINATION ET PARTAGE DU POUVOIR: LE CAS DES ÉPONYMES
}

\author{
Uzoma CHUKwU \\ Centre de Recherche en Terminologie et Traduction, Université Lumière, Lyon, France
}

\begin{abstract}
Résumé
Si le recours à l'éponyme est un procédé de formation de termes très productif dans certains domaines, il reste néanmoins un phénomène très peu étudié par les linguistes et, plus particulièrement, les terminologues qui semblent renvoyer la balle à la logique. D'autre part, à en juger par les invectives fréquentes contre les éponymes, ils n'ont, dans l'ensemble, pas bonne presse chez les utilisateurs de la langue. Cependant, envisagée par rapport à des domaines particuliers, la situation paraît beaucoup plus nuancée. En médecine, par exemple, ils rencontrent beaucoup d'hostilité parce qu'ils sont perçus comme des facteurs perturbateurs de la terminologie du domaine, pour l'essentiel transparente puisque largement fondée sur la composition savante. En histoire naturelle, ils sont plutôt bien intégrés et forment même l'ossature du système binominal de Linné, alors qu'en physique let en mathématiques), ils ne semblent pas susciter de sentiment particulier.
\end{abstract}

Mais si le procédé se maintient dans ce climat a priori défavorable, c'est d'abord parce qu'il étend considérablement la capacité de dénomination des langues naturelles en mettant à leur disposition tout le répertoire des patronymes en nombre quasi illimité. Son maintien s'explique aussi par le fait qu'il participe au processus de régulation interne de la science en permettant à celle-ci de reconnaître les mérites des siens.

\begin{abstract}
Although eponymy has continued to be very productive in the terminology of some domains, it has yet to merit the attention of linguists and, in particular, terminologists who seem to consider it as properly belonging to the realm of logic, much like the proper noun. Furthermore, the general impression of an attitude of near-hostility of language users toward eponymous terms contrasts with the domain-specific welcome that has been reserved for them. In medicine, for example, eponyms have often been greeted with hostility because they are perceived as a destabilising factor in medical terminology, which is in the main based on composition and, therefore, offers some measure of ready intelligibility. In natural history they seem quite at home in the binomial system popularized by Linnaeus whereas in physics (and mathematics) the situation is one of indifference.
\end{abstract}

However, despite what one may still consider as a generally unfavourable climate, eponymy will continue to maintain itself for at least two reasons. First, it significantly extends the naming capacity of natural languages. Second, it plays a central role in the regulatory process of science by providing a means for sharing the spoils of scientific enterprise.

\section{INTRODUCTION}

À en juger par la pauvreté de la bibliographie consacrée au type de dénomination qui consiste à donner un nom propre à un phénomène, un concept ou un objet scientifique ou technique, on pourrait croire qu'il s'agit d'un procédé marginal. Or, les faits semblent suggérer le contraire et si on se fie au seul critère statistique, on constate que le phénomène occupe une place importante dans les techniques de formation de termes dans 
certains domaines. Des dictionnaires entiers sont d'ailleurs consacrés au recensement et à la description de termes issus de ce procédé. Il est d'autre part intéressant de constater que le seul type de travaux consacrés entièrement au phénomène relève essentiellement du recensement. Nous pensons ici non seulement aux dictionnaires d'éponymes à proprement parler, comme ceux consultés pour les besoins de la présente étude, mais aussi au seul article qui leur est exclusivement consacré (voir van Hoof 1986). En dehors de la note liminaire et de la tentative de classement selon le type de problème de traduction posé, cet article reste essentiellement un travail de recensement.

La présente étude en tentera donc une première analyse. Dans un premier temps, nous essaierons d'expliquer le manque d'intérêt des linguistes et, plus particulièrement, des terminologues pour le procédé. Il nous semble qu'une meilleure compréhension de l'attitude de ceux-ci envers le phénomène offre un premier axe pour mieux saisir sa nature et son fonctionnement. Ensuite, nous examinerons l'accueil réservé aux éponymes par les utilisateurs de la langue en nous appuyant sur l'exemple de quelques domaines (la médecine, l'histoire naturelle et la physique) et situations d'utilisation (la traduction). On tentera également d'expliquer le maintien du procédé malgré des conditions d'accueil que nous qualifierons d'emblée de difficiles. Cela nous amènera à situer le procédé dans le double processus de régulation de la communauté scientifique et de constitution de la connaissance scientifique.

\section{LES TERMINOLOGUES FACE À L'ÉPONYME}

Si l'on s'interroge sur l'absence de réflexions des linguistes et, en particulier, des terminologues consacrées aux éponymes, il convient de chercher d'abord du côté de la logique qui sous-tend le phénomène. Certes, les éponymes font partie de l'ensemble des termes dont dispose un domaine scientifique ou technique pour désigner les objets et concepts manipulés et sont donc des objets linguistiques. Cependant, ils laissent l'impression d'obéir à une autre logique, celle du nom propre qu'ils reprennent, tel quel ou aménagé (voir Kleiber, dans ce volume). À ce titre, leur étude relèverait de l'analyse philosophique, car ils sont du domaine de la référence unique, ne nécessitant pas le même type de médiatisation psychologique qu'on suppose à l'œuvre dans les autres signes linguistiques entre réalité linguistique (son ou graphie) et réalité extralinguistique (la chose dénommée).

Un tel raisonnement est toutefois facilement pris en défaut. D'une part, si on l'examine de près, il ne fait que souscrire à un a priori, à savoir que l'éponyme hérite forcément des problèmes d'analyse du nom propre dont il est issu et que, par conséquent, les questions habituelles posées sur le rapport entre signifiant et signifié cessent d'être pertinentes. D'autre part, il ne semble pas que le principe de l'arbitraire du signe ait été accepté, en tout cas en terminologie, pour ce qu'il est, c'est-à-dire un principe, un postulat théoriquement non vérifiable. L'analyse terminologique semble de plus en plus chercher dans les termes «un signe des choses», comme le démontrent bien les contributions (dans ce volume) de Boisson sur les dénominations de la règle à calcul et de Thoiron et al. sur la construction de l'archi-concept.

Le faible intérêt porté à la question des éponymes par les terminologues tient aussi aux exigences du nouveau programme d'une terminologie «science» des systèmes formés par l'ensemble des termes de domaines spécifiques. L'abandon progressif d'une vision énumérative (nomenclature) de la terminologie en faveur d'une vision taxinomique s'accommode mal de cette catégorie de termes'. Tout au plus un système énumératif, comme celui que constitueraient les éponymes d'un domaine donné, se prêterait-il au recensement et à la description des items individuels, mais il ne révélera aucune structure 
permettant le classement onomasiologique et toute l'analyse à laquelle celui-ci peut donner lieu et dont rêve tout terminologue. Le fait que les terminologues n'aient pas songé à donner un nom au procédé en dit long sur leur attitude vis-à-vis de celui-ci.

Un autre problème majeur avec les éponymes concerne l'absence de paradigmes formels cohérents, même à l'intérieur d'un même domaine. Des formes dans lesquelles le patronyme est repris tel quel en côtoient d'autres construites avec le génitif ou le possessif, ou d'autres avec adjectivation ou substantivation du patronyme ou, parfois, une combinaison de deux transformations comme dans «Tutle's Hamiltonian theorem» ${ }^{2}$ sans qu'on puisse dire pourquoi. Pourquoi est-ce qu'on dit «Fermat's equation» et «Einstein's equation for specific heat» mais plutôt «Einstein equations»? Pourquoi dit-on «Schwarz function» mais plutôt «Schwarzian derivative»? Qu'en est-il des éponymes composés ? Si «Maxwell-Boltzmann equation» est bien l'équation de Maxwell et Boltzmann, «EwaldKornfeld method» est une extension de «Ewald method» en physique des solides et «Bohr-Sommerfield theory» une modification de «Bohr theory». Si «Bessel transform» est synonyme de «Bessel-Fourier transform», «Boltzmann equation» n'est pas synonyme de «Maxwell-Boltzmann equation ${ }^{3}$. Parfois, les personnages qui prêtent leur patronyme aux appellations éponymiques composées sont co-auteurs de la publication dans laquelle a été mis en évidence le phénomène pour la première fois; parfois, il s'agit d'auteurs successifs. Ce dernier cas illustré par «syndrome de Stokes-Adams», qui pose par ailleurs un problème supplémentaire: alors que la première description du syndrome fut faite par Adams en 1827, dans l'éponyme consacré, c'est Stokes (dont la description date seulement de 1846) qui précède Adams4.

\section{ACCUEIL DES ÉPONYMES PAR LES UTILISATEURS DE LA LANGUE}

Il faut reconnaître que dans l'ensemble, les éponymes n'ont pas rencontré un accueil très favorable chez les utilisateurs de la langue, en ce sens qu'ils sont globalement perçus comme des éléments perturbateurs de systèmes terminologiques (voir, par exemple, Dirckx 1983, cité plus loin). Mais ce jugement d'ensemble cache une réalité beaucoup plus nuancée qui oblige à poser le problème par rapport à des domaines bien précis. À ce propos, on peut considérer les trois grands domaines de la médecine, de I'histoire naturelle et de la physique (avec les mathématiques ${ }^{6}$ ) comme faisant partie de ceux dans lesquels le recours à l'éponyme est très répandu. Or, par un concours de circonstances extraordinaire, la situation dans ces trois grands domaines reflète assez bien aussi les trois grandes tendances en la matière. Nous examinerons donc de près dans le reste de cette section l'accueil réservé aux éponymes dans ces trois domaines avant de poser le problème par rapport à la traduction.

\subsection{LES ÉPONYMES EN MÉDECINE}

Pour la médecine, on peut dire que l'accueil n'a pas été du tout chaleureux malgré la très grande place qu'y occupe le procédé6. Ceci ne devrait toutefois pas tellement étonner quand on considère la source à laquelle s'alimente pour l'essentiel la terminologie médicale et la pratique dominante en matière de dénomination dans le domaine. La médecine est un domaine dont l'histoire se confond avec celle de l'humanité qui, au cours du temps, a pu se doter d'un système de formation de termes basé sur la combinatoire d'éléments issus de langues anciennes (principalement le grec et le latin) et dont le grand mérite est la systématicité et la transparence des unités ainsi formées. Ainsi, tout initié devrait pouvoir décoder un nouveau terme rencontré s'il est formé dans les règles avec les formants gréco-latins habituels. Quand on considère le nombre de termes que le médecin moyen doit maîtriser, l'avantage de ce système paraît évident. Car si les éponymes font 
appel à la seule mémorisation (puisque chaque terme doit être appris et mémorisé avec son sens), une terminologie systématique du type de celle permise par les termes savants permet le stockage et la manipulation de règles de décodage dont le nombre est forcément réduit, diminuant ainsi la charge mémorielle de l'utilisateur.

Il suffit pour s'en convaincre de comparer, par exemple, le terme éponymique «maladie nerveuse de Recklinghausen» à son synonyme «neurofibromatose», bâti avec des formants savants. Avec le terme éponymique, le médecin saura qu'il s'agit d'une maladie nerveuse, information sans doute pertinente mais insuffisante quand on sait qu'il y en a des centaines?. En revanche, l'analyse de son synonyme bâti avec des formants savants conduit facilement à la glose: «maladie affectant les fibres reliant les différentes parties du système nerveux».

On peut donc considérer que si le phénomène a si mauvaise presse dans le domaine de la médecine, c'est parce qu'il constitue un élément perturbateur de l'ordre qui est recherché dans la terminologie du domaine, essentiellement fondée sur la composition savante. C'est le sentiment que nous laisse Dirckx (1983) dans les deux derniers paragraphes de la section de son ouvrage consacrée aux éponymes:

The most telling objection to the continued manufacture of medical eponyms is not the difficulty of pronouncing Chvostek [...] A more troublesome feature of eponymy is that it produces lexically empty terms-words that supply no clue to their reference or bearing. Quite apart from the needless trouble that this causes the learner, it runs directly counter to the spirit of scientific terminology, which strives for order, consistency, and at least some measure of ready intelligibility.

[...] Given two eponyms, one has no way of knowing whether they refer to related concepts, are synonymous, or have no logical association whatever. (Dirckx 1983:84)

\subsection{LES ÉPONYMES EN HISTOIRE NATURELLE}

S'agissant de la situation en histoire naturelle, qui est le domaine où l'on a le plus recours à ce procédé de dénomination et de loin, l'éponyme ne semble pas du tout constituer une menace pour un ordre quelconque, et des règles ont été définies pour régir la formation de termes éponymiques. Les codes de nomenclature dans les différentes disciplines de ce domaine définissent des règles claires d'intégration du procédé dans le système binaire adopté depuis Linné : règles de latinisation des noms, de désinence (distinction entre masculin et féminin), règles d'orthographe, mais aussi règles régissant la signalisation de création successive d'espèces et même de création anonyme, les espèces telles qu'elles sont désignées n'existant pas dans la nature en tant que telles (voir Pajaud 1989 et Blackwelder 1967 pour ces règles). Pourtant, il serait erroné de chercher à expliquer les heurs de l'éponyme dans ce domaine par cette organisation de son emploi : ce serait prendre l'effet pour la cause. Nous pensons plutôt que l'histoire naturelle est le domaine de l'énumératif (la nomenclature par excellence), en ce sens qu'elle décrit les espèces naturelles dont les noms s'accommodent bien du système de la référence unique que constitue l'éponyme. Car le nom de l'espèce en histoire naturelle est en réalité celui de l'individu type : «À l'individu-type - quel que soit le concept d'espèce sous lequel il sera un jour rangé par la Science, au cours du changement inévitable des conceptions taxinomiques - le nom de l'espèce est indissolublement attaché et, à travers lui, les noms de toutes les unités supérieures» (Richter cité dans Pajaud 1989: 47). Contrairement, donc, à ce qu'on pourrait croire de par son expérience avec la désignation dans d'autres domaines scientifiques, la recherche de stabilité des noms de genres et d'espèces en histoire naturelle n'est pas basée sur les définitions (en tout cas, cette prétention a été 
abandonnée au début de ce siècle), comme dans la plupart des autres systèmes terminologiques.

\subsection{LES ÉPONYMES EN PHYSIQUE}

Pour la physique (et les mathématiques dont elle partage beaucoup de notions ${ }^{8}$ ), on peut résumer la situation en deux mots: absence d'ordre et indifférence, la même indifférence qui caractérise l'attitude des physiciens vis-à-vis de l'ensemble de la terminologie de leur discipline. Cela est tout à fait compréhensible, car il est difficile de voir en quoi «diffusion élastique», terme non éponymique de physique, se prêterait plus au type d'analyse qui intéresse la terminologie que «diffusion de Rutherford», terme éponymique du même domaine. C'est un domaine où l'on ne s'est jamais préoccupé de terminologie, en tout cas pas dans un grand effort de régulation d'ensemble comparable à celui qui a été fait pour la chimie (cf. les efforts de réforme de la terminologie de la chimie à la fin du XVIII' siècle qui ont associé des noms comme Guyton de Morveau et Lavoisier).

La terminologie de la physique puise beaucoup dans la langue générale, dont les mots sont repris et souvent affectés de sens nouveaux, ce qui peut parfois constituer un piège pour le profane. À titre d'exemple, le terminologue qui se fonderait dans le repérage des termes sur l'heuristique selon laquelle les termes scientifiques prennent souvent des formes qui ne sont pas courantes dans la langue générale - heuristique qui semble efficace pour bon nombre de domaines scientifiques - passera à côté de termes comme: «aberration», «charme», «efficacité», «liberté», etc. Si on ne peut pas dire que la terminologie du domaine constitue un système nomenclatural, on ne peut pas dire non plus qu'elle se prête à la systématisation et la structuration associées aux systèmes taxinomiques $^{9}$. Le travail d'apprentissage de la terminologie du domaine est donc essentiellement un travail de mémorisation, comme pour les systèmes nomenclaturaux. On peut donc penser qu'il n'y a pas d'ordre pour lequel le recours à l'éponyme aurait constitué une menace.

\subsection{LES ÉPONYMES EN TRADUCTION}

Le traducteur n'est pas un utilisateur de la langue du même type que le physicien ou le médecin, mais une discussion terminologique sur l'éponyme ne peut éviter d'évoquer les problèmes qu'il pose dans des situations de traduction. D'une part, historiquement, le développement de la terminologie a eu pour moteur principal la traduction. D'autre part, le rôle d'interface que joue le traducteur entre les langues en contact le place au cœur de la problématique de l'éponyme. En dehors des problèmes associés à la variabilité de la forme de l'éponyme au sein d'une même langue (comme on le verra plus loin), le traducteur doit aussi gérer ceux de l'imprévisibilité de la forme de l'éponyme d'une langue à l'autre (voir le classement de van Hoof (1986) établi en fonction du problème de traduction particulier posé). Plus que tout autre utilisateur de la langue, le traducteur doit donc mettre à rude épreuve sa mémoire pour retenir les termes éponymiques et leurs équivalents en langue d'arrivée ou, à défaut, consulter sans cesse des dictionnaires. Assez souvent, à cause des facteurs affectifs liés à l'adoption et l'emploi effectif de l'éponyme (le rédacteur de dictionnaire ayant naturellement tendance à ne recenser que les éponymes qui ont cours «chez lui»), ces dictionnaires peuvent ne pas être d'un grand secours, non seulement du point de vue des éponymes retenus dans leur nomenclature, mais aussi du point de vue du traitement micro-structural de ceux-cilo. Tous ces problèmes augmentent la pression sur le temps psychologiquell chez le traducteur, ce qui ne peut que porter atteinte à la qualité de son travail et le pousser à déplorer le procédé de l'éponymie. 


\section{UN PHÉNOMÈNE DE MODE QUI N'EN EST PAS UN}

Le recours à l'éponyme comme procédé de formation de termes a souvent été considéré comme un phénomène lié à la mode, donc un phénomène passager ${ }^{12}$. Or, la réalité dans les disciplines qui en usent semble indiquer le contraire. Il faut donc se demander pourquoi un phénomène qui semble inspirer l'indifférence, voire l'hostilité, continue d'être productif. Car même si des cas d'appellations éponymiques qui n'ont pas résisté au temps sont connus, le procédé lui-même résiste bien. Comme on l'a déjà vu, pour certains domaines (dont surtout l'histoire naturelle), on peut supposer que le procédé est bien adapté à la réalité étudiée, alors que pour d'autres (la physique et les mathématiques), l'éponyme ne semble pas poser plus de problèmes que d'autres catégories de termes. Cependant, si l'on tient à comprendre les raisons du maintien du procédé pour l'ensemble des sciences et techniques, il faudra chercher ailleurs, en particulier dans le mode de régulation des communautés scientifiques.

\section{1. ÉPONYMES ET BESOINS ILLIMITÉS DE DÉSIGNATION DANS LES SCIENCES \\ ET LES TECHNIQUES}

Il faut reconnaître que si le recours à l'éponyme se porte bien, c'est bien parce qu'il offre des possibilités de dénomination que d'autres procédés n'offrent pas. Les éponymes étendent considérablement les capacités de dénomination des langues naturelles en mettant à leur disposition tout le répertoire des patronymes en nombre quasi illimité, car derrière toute appellation éponymique, il y a un état civil et un curriculum vitce ${ }^{13}$. C'est un atout dont on ne peut mesurer l'avantage qu'en considérant le recours à ce procédé par rapport à des domaines comme ceux de l'histoire naturelle ou de la physique (et des mathématiques). Aucun système de dénomination raisonné14 ne saurait générer des termes pour, par exemple, toutes les notions mathématiques connues et actuellement désignées par des combinaisons «relation + nom propre» (avec leurs nombreuses transformations), compte tenu surtout de la source essentiellement vernaculaire de la terminologie mathématique. On peut en dire autant des noms des espèces dans l'ensemble des disciplines de l'histoire naturelle. Il y a donc là raison de soutenir que si le procédé se maintient bien, c'est parce que la terminologie a horreur du vide et que seul ce procédé offre des possibilités en nombre quasi illimité de remplir ce vide.

De même, si l'on reproche à l'éponyme d'augmenter la charge mémorielle de l'utilisateur de la langue, on y a aussi parfois recours pour les mêmes raisons qui expliquent le passage du terme-syntagme au sigle, à savoir le soulagement de la mémoire de l'utilisateur. Ici, il faut se rappeler que dans certains domaines, dont surtout la médecine, le recours à l'éponyme s'explique parfois par le désir de court-circuiter des termessyntagmes descriptifs considérés trop longs (Hamburger 1982) et donc posant des problèmes de mémorisation et de mise en discours. La comparaison de l'éponyme «syndrome de Moschowitz» avec son synonyme non éponymique «pupira thrombotique thrombocytopénique» ou celle de «syndrome de Barter» avec son synonyme «hypokaliémie chronique familiale avec hyperkaliurie», cité par Hamburger (1982), est assez parlante à cet égard. On peut aussi supposer que la même motivation est à l'œuvre dans le couple «maladie de Takayashu» et «maladie des femmes sans pouls», encore qu'une analyse trop moderne ne craignant pas d'être taxée d'anachronisme pourrait conduire à y voir aussi la recherche du politiquement correct ${ }^{15}$.

\section{2. ÉPONYMES DANS LE MÉCANISME DE RÉGULATION DES COMMUNAUTÉS SCIENTIFIQUES}

Ziman (1984), entre autres, distingue une sociologie externe de la science, qui concernerait les rapports de la communauté scientifique avec la société dont elle dépend, et une sociologie interne de la science, qui concernerait les modes de régulation au sein 
de la communauté scientifique. Une communauté qui n'aurait pas de règles (même implicites) régissant le partage des rôles et des mérites, et donc du pouvoir, ne saurait motiver les siens à donner le meilleur d'eux-mêmes. De toute évidence, le recours à l'éponyme comme procédé de dénomination s'inscrirait dans le cadre de cette régulation des rapports entre les acteurs de la communauté scientifique, au même titre que les prix scientifiques les plus valorisés ou même que la signature d'un article ${ }^{16}$. L'éponyme paraît donc a priori comme un moyen permettant de reconnaître les mérites de ceux dont la contribution a été particulièrement importante pour l'avancement d'une science ou d'une technique.

Il faut toutefois s'empresser d'ajouter que si dans la plupart des cas il s'agit de désigner un phénomène ou un objet par le nom du scientifique qui l'a décrit ou découvert, il y a aussi des cas où le personnage du patronyme est bien un homme de science mais qui n'est pas lié directement au phénomène ou à l'objet désigné, ce qui n'est pas sans poser de sérieux problèmes à l'historien des sciences (voir plus loin). Nombreux sont les cas où le choix du patronyme d'une appellation éponymique s'inscrit plutôt dans une logique où l'on cherche à rendre hommage à un scientifique qui a contribué à l'avancement de la science dans d'autres domaines. Sous l'entrée «Lindbergh pump» (appelé aussi «Carrel-Lindbergh pump»), nom de l'appareil de perfusion utilisé pour maintenir les organes en vie hors du corps, le Academic Press Dictionary of Science and Technology indique que l'éponyme a été choisi en hommage à l'aviateur américain Charles Lindbergh. De la même façon, l'espèce «Glazewskia demarcqii» est ainsi dénommée par son fondateur en hommage à un certain Monsieur Gérard Demarcq, alors que «Moravamylacris kiukolovec» est ainsi dénommée en hommage à Madame Jarmila Kukalova (voir Pajaud 1989: 33) alors que la pratique dominante en histoire naturelle est d'utiliser le patronyme du fondateur de l'espèce.

Dans d'autres cas, le personnage qui a prêté son patronyme à l'appellation peut ne pas avoir de rapport direct ni avec le phénomène ni avec la science de façon générale. Dans le recensement des cent appellations éponymiques à retenir par le médecin présenté dans Hamburger (1982: 148-149) il est fait mention d'un «syndrome de Pickwick» dont «Le Monsieur Pickwick patronyme [... du] syndrome est le célèbre personnage de Dickens, dont la silhouette évoque celle des malades, obèses, gros mangeurs, toujours somnolents, un peu asphyxiques, avec tendance à la polyglobulie et à l'insuffisance ventriculaire droite» 17 .

Des cas sont également connus où c'est le nom du patient chez qui la maladie a été décrite qui sert de patronyme à l'appellation (voir l'exemple de Morbus Mortimer, qui a donné son nom à la maladie de peau, la sarcoïdose, décrite par Hutchinson en 1908). Un autre cas intéressant en anglais est celui de «Christmas disease», désignant l'hémophilie B et qui, de prime abord, fait penser à la fête de Noël, mais dont le patronyme repris dans l'éponyme est celui du patient chez qui la maladie a été décrite le plus en détail. Firkin \& Whitworth (1987) citent aussi quatre exemples d'appellations éponymiques dont les origines restent inexpliquées: «Behçet disease» décrite par Bluthe, «Osler-Rendu-Weber telangiectasia» décrite par Babington, «Reiter syndrome» décrite par Brodie et «Kartagener syndrome» décrite par le Russe Sievert. On en conclut donc que la règle générale en la matière peut parfois cacher une réalité beaucoup plus complexe.

Il est également courant d'avoir recours au nom de personnages mythiques. La malformation appelée «hermaphrodisme» serait ainsi nommée en l'《honneur» du fils d'Hermès et Aphrodite dont le corps se serait fusionné avec celui d'une nymphe pour donner un mélange de caractéristiques physiques masculines et féminines. Il y a aussi «Rhea», en astronomie qui emprunte le nom du Titan de la mythologie grecque, qui est à la fois soeur et épouse de Cronus qui, lui-même, est connu dans la même mythologie pour 
avoir dévoré sa progéniture, ce qui explique l'utilisation de son nom dans le terme «cronisme», qui désigne en psychologie ce comportement aberrant. Ce type d'éponymes est très usité dans les sciences du comportement en général (cf. les complexes d'CEdipe et d'Électre), car les personnages de la mythologie grecque illustrent souvent un comporternent avec lequel ils finissent par se confondre. Les noms des corps célestes s'alimentent aussi à cette source, bien que les raisons en soient différentes.

Il arrive aussi que le nom utilisé dans l'appellation éponymique ne soit pas celui d'une personne ${ }^{18}$. On trouve, par exemple, les noms de lieu: «fièvre exanthématique de l'Est africain», «St. Gotthard's tunnel disease», «fièvre de Queensland», «Coxsackievirus» (appelé ainsi parce que le virus fut isolé pour la première fois en $1950 \mathrm{chez}$ un patient à Coxsackie, New York), en médecine. La pratique est courante en histoire naturelle, surtout lorsqu'il s'agit de marquer l'empreinte de la provenance de l'espèce désignée ou sa répartition géographique («Latimeria chalumnce», nom de l'espèce de colacanthe pêchée dans le canal de Mozambique, à l'embouchure de la rivière Chalumna (Pajaud 1989)). Le nom utilisé dans l'appellation éponymique peut aussi être celui d'une unité stratigraphique, encore qu'on ne sache pas trop parfois s'il s'agit vraiment de nom d'unités stratigraphiques ou de nom de lieux géographiques, étant donné la relation de dérivation entre les premiers et les noms latins de certaines régions.

Pour revenir à l'éponyme en bonne et due forme, notons que la reconnaissance du scientifique individuel qu'il permet va à contre-courant du mode d'organisation de la recherche scientifique moderne. Autant la science de l'Antiquité et des périodes précédant la nôtre était caractérisée par la quête individuelle se confondant tantôt avec la quête philosophique, tantôt avec la quête de la sublimation artistique (le cas de Léonard de Vinci en est la parfaite illustration), autant la science moderne est caractérisée par le travail d'équipe nécessitant souvent la synthèse de compétences diverses. Il y a donc là une source de tension qu'on pourrait tenter d'expliquer en regardant du côté des textes produits par la science.

Ailleurs (Chukwu 1993), nous établissions une distinction entre discours technique et discours scientifique, distinction qui repose essentiellement sur la responsabilité du scripteur vis-à-vis de son texte. Nous attirions en particulier l'attention sur le fait qu'alors que le discours technique est un discours donné tel quel, sans garantie quelconque quant à sa valeur (un discours qui admet même l'anonymat), le discours scientifique requiert la signature d'une personne physique, garante de sa valeur, de son sérieux, sans quoi la communauté scientifique ne va même pas se donner la peine de passer une telle contribution au crible19. Même si l'organisme d'affiliation du scientifique, la communauté scientifique nationale et la nation tout entière s'honorent des percées scientifiques permises par l'un des leurs, quand il s'agit de déterminer les responsabilités en cas d'erreur ou de fraude, le scientifique se retrouve seul face à ses juges (cf. l'affaire de la mémoire de l'eau). Le recours à l'éponyme paraît donc répondre à la double logique du «rendre à César ce qui est à César» et de la désignation de responsables: l'un est forcément le pendant de l'autre.

L'histoire de la découverte, de la validation et du développement des rayons $\mathrm{X}$ comme technique incontoumable de l'investigation médicale, ainsi qu'elle est reconstituée par Sterpellone (1986), mérite un rappel ici, car elle est un classique du mode de régulation interne de la science et du rôle que l'éponyme peut y jouer. Rappelons-en les grandes lignes. Le 8 novembre 1895, Wilhelm Conrad Röntgen découvre tout à fait par hasard des rayons inconnus produits lorsque les rayons cathodiques touchent l'intérieur d'un tube en verre (en l'occurrence le tube de Crooke, ancêtre du tube à vide), rayons qu'il allait par la suite appeler «rayons $X »$, avec le même $X$ utilisé en mathématiques lorsqu'il s'agit d'une quantité inconnue. Réplication du phénomène pour s'assurer de sa 
reproductibilité et des conditions de cette reproductibilité. Établissement de la preuve de l'existence des rayons $X$ en photographiant la main gauche de sa femme avec alliance, photographie considérée comme le premier röntgenogramme de l'histoire de la radiologie. Rédaction d'une communication préliminaire sur la découverte pour la Société de physique médicale de Würzburg avec l'intention de la faire dater, même de quelques jours seulement, de l'année 1895 . Le 28 décembre 1895 , présentation de la première communication publique, intitulée Eine neue Art von Strahlen (Un nouveau type de rayons), par

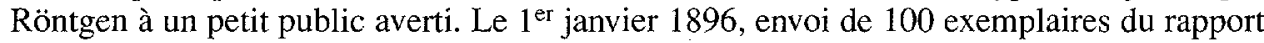
et des radiographies à autant de collègues et d'experts.

Dès janvier 1896, le relais de diffusion par la presse populaire et à sensation partout dans le monde permet à la découverte de sortir vite du laboratoire et des revues spécialisées pour rechercher cette autre validation qu'est l'utilité des découvertes scientifiques pour le grand public. Naturellement, l'accaparement par la presse populaire comporte ses risques: très peu de découvertes scientifiques auront donné lieu aux mythes et fantasmes suscités par la découverte de Röntgen, non seulement parmi les profanes et autres amateurs de voyeurisme, qui se délectaient à la perspective de tout ce qu'ils allaient pouvoir en faire, mais aussi parmi les scientifiques ${ }^{20}$.

Vient ensuite la première reconnaissance: l'invitation du professeur Röntgen par l'empereur Guillaume II pour que celui-ci (désormais devenu le professeur von Röntgen) refasse ses expériences devant un public d'hommes d'État et de science. Vinrent ensuite l'apparition d'éponymes ${ }^{21}$ empruntant le patronyme de Röntgen et finalement (en 1901) le prix Nobel de physique «pour une découverte qui a beaucoup apporté à l'humanité». Toute cette gloire n'a pas manqué de créer des jaloux et de tous les continents affluaient des revendications et contre-revendications pour une part du «gâteau» ou, à défaut, pour la reconnaissance de travaux non publiés qui auraient préparé la découverte de Röntgen. La plus virulente de ces revendications étant celle du professeur Philipp Lenard, établi en Allemagne mais d'origine austro-hongroise, qui a comparé Röntgen à la sage-femme qui, même si elle est le premier à montrer le nouveau-né, ne peut en aucun cas être prise pour la mère. Lenard a même fait jouer ses relations nazies pour mettre en avant ses revendications et ce n'est qu'en 1951 que l'Allemagne fédérale a rectifié les méfaits nazis sur la question en sortant un timbre avec l'effigie de Röntgen. Aujourd'hui, on peut considérer que, plus que le prix Nobel (le professeur Philipp Lenard ayant été lui-même prix Nobel de physique), les quatorze termes éponymiques honorant Röntgen ne laissent aucun doute sur l'identité du vainqueur de la bataille pour la paternité de la découverte des rayons $X$.

La question qui se pose après ces longues péripéties est celle de savoir qui décide du recours à l'éponyme. C'est une question qui n'est pas sans pertinence, puisque si ce sont d'autres scientifiques, comme on peut le supposer dans beaucoup de cas, il s'agira avant tout de la reconnaissance du scientifique par ses pairs. En revanche, s'il s'agit du scientifique donnant son nom à un phénomène, concept ou objet (ce qu'on peut supposer rare sauf dans les nomenclatures bionaturalistes lorsqu'il s'agit de donner le nom du créateur d'une espèce), il s'agira plutôt d'auto-reconnaissance et le processus s'inscrirait dans le cadre de la lutte pour le partage du pouvoir scientifique. Dans un cas comme dans l'autre, de nombreux problèmes sont soulevés. Dans le premier cas, il existe le risque d'attribuer au scientifique une idée qui n'est pas vraiment la sienne avec tous les problèmes d'engagement de responsabilité que cela suppose 22 . On l'a vu en linguistique avec la remise en question des idées sur le langage et le réel soutenues sous le couvert de l'hypothèse de Sapir-Whorf et tout le débat sur la responsabilité de Whorf. Aujourd'hui, il semble même qu'on soit obligé de revoir une bonne partie de tout ce qui a été transmis sous cette appellation, puisque, selon Alford (1995), une bonne partie de la littérature sur cette question serait même aux antipodes de ce que Whorf aurait écrit sur un phénomène 
qu'il avait préféré appeler, en connaissance de cause, le principe de relativité linguistique $^{23}$. On voit ici les limites du recours à l'éponyme. En liant trop étroitement un concept au nom d'une autorité, l'éponyme donne facilement lieu à la dérive qui consiste à tout justifier au nom de cette autorité.

Dans le second cas, la motivation dominante est clairement celle de la lutte pour le pouvoir scientifique. Non pas qu'on puisse entièrement écarter une telle hypothèse dans le premier cas, puisqu'il s'agit aussi de «qui reçoit quoi» 24 . Cependant, en soi, cela ne nous paraît pas anormal, mais si le problème est soulevé ici, c'est parce qu'il permet d'expliquer le fait que parfois le patronyme emprunté par l'appellation éponymique change d'un pays à l'autre, d'un continent à l'autre et même d'une région (ou laboratoire) à une autre du même pays. À ce propos, dans le classement établi par van Hoof (1986), on trouve au moins 29 cas de changement de patronyme entre le français et l'anglais, sans compter les nombreux cas d'introduction d'un ou de plusieurs patronymes supplémentaires ou d'abandon de l'appellation éponymique dans l'une des deux langues. En matière de synonymie d'éponymes, il dénombre pour la seule langue anglaise au moins 84 cas, dont surtout «Clarke's column», appelé aussi «Gierke's bundle», «Krause's bundle» et «Stilling's column». Dans l'introduction à leur dictionnaire d'éponymes médicaux, Firkin \& Whitworth (1987) citent quelques exemples très instructifs. Le premier est celui du cholécystogramme appelé «cholecystogram» à Melbourne et «Graham test» à Sydney en Australie. L'autre exemple est celui de la maladie appelée «Plummer-Vinson syndrome» aux États-Unis et en Australie (patronymes retenus aussi dans l'éponyme français) mais «Patterson-Kelly syndrome» au Royaume Uni et «Waldenström-Kjellberg syndrome» dans les pays scandinaves. Dans ce dernier exemple, il est peut-être à propos de souligner que l'origine des scientifiques dont les patronymes sont repris dans les appellations n'est pas sans rapport avec cette synonymie géographique. Le recours aux éponymes et le choix de ceux-ci peuvent aussi traduire la concurrence sans merci entre les différentes recherches nationales ${ }^{25}$ ou des «niches» de recherche (qui peuvent transcender des frontières nationales) ou des laboratoires de recherche travaillant dans un même secteur.

\section{3. ÉPONYMES ET HISTOIRE DES SCIENCES}

On cherche aussi souvent dans les éponymes des jalons marquant des étapes importantes du progrès de la science, des jalons qui devraient permettre à l'historien des sciences de se retrouver dans l'immense enchevêtrement d'efforts individuels qu'est la science. Firkin \& Whitworth (1987) soutiennent, en particulier pour le cas des appellations éponymiques médicales, que l'étude de celles-ci avec leur datation devrait permettre de constater comment les grandes nations de la recherche médicale se sont passé le relais: de l'Italie jusqu'aux États-Unis en passant par la France, l'Angleterre, l'Autriche et l'Allemagne.

Paradoxalement, on peut tout aussi légitimement soutenir que l'utilisation de l'éponyme dans ce but nie le caractère fondamentalement composite et historique de la connaissance scientifique qui se précise, parce que des générations successives de scientifiques se relayent pour la faire avancer. Ainsi, parler de «loi de Bode», c'est peut-être oublier trop vite qu'il fallait que Johann Daniel Titius (1729-1796) attire l'attention de ses pairs sur la relation arithmétique simple entre les distances entre les soleils des planètes connues pour que Johann Elert Bode (1747-1826) puisse penser à rendre 1'idée populaire; c'est créer les conditions du passage au second plan de tout le travail accompli par des générations successives de scientifiques dans la vérification de la loi (William Herschel, Franz Xavier von Zach, Giuseppe Piazzi, H. Olbers, John Couch Adams et Urbain Jean Joseph Le Verrier) jusqu'à la découverte de Neptune dont la distance par rapport au Soleil s'est révélée différente et a donc entraîné le discrédit de la loi (voir Bynum, Browne \& 
Porter 1983)26. Mais si nous avons voulu aller jusqu'au bout de cet exemple, c'est aussi pour souligner la constante dialectique entre vénération et iconoclasme vis-à-vis de l'autorité scientifique dans le mode de régulation de la science ${ }^{27}$. Car, même en se prêtant à ce jeu de partage de pouvoir, aucun scientifique n'est dupe: «Truth is the daughter of time and not of authority» (Francis Bacon, cité en épigraphe de Firkin \& Whithorth 1987).

\section{CONCLUSION}

En guise de conclusion, interrogeons-nous sur l'avenir du recours à l'éponyme comme procédé de dénomination: va-t-il se maintenir, ou est-il appelé à disparaître? La variété des attitudes à son égard selon les domaines incite à la prudence dans la formulation de toute prédiction. Cependant, on peut penser que, en tant que procédé de formation de termes, le recours à l'éponyme se maintiendra sans problème dans deux des trois grands domaines qui ont, pour l'essentiel, servi de base à nos discussions ci-dessus, à savoir l'histoire naturelle et la physique (et les mathématiques). Les raisons en sont multiples, comme on l'a vu, et vont de l'adaptation de l'éponymie à la réalité étudiée par le domaine (notamment pour l'histoire naturelle) à l'indifférence des physiciens qui n'ont jamais cru utile de poser le problème de la terminologie de leur domaine.

Reste la question de l'avenir du procédé dans des domaines où le procédé est très productif mais où, en même temps les éponymes n'ont jamais eu bonne presse, comme la médecine. Faut-il croire à l'abandon progressif du procédé, comme le prédisent Hamburger (1982) et Dirckx (1983)? L'analyse que nous avons faite ici de la problématique des éponymes nous interdit d'envisager une telle perspective. Car, au-delà de leur fonction de dénomination, nous avons vu que les éponymes remplissaient également une fonction psychosociologique en science: ils permettent à la science non seulement de reconnaître les siens, mais aussi de les inciter à donner le meilleur d'eux-mêmes. En revanche, nous pensons que la tendance vers le développement de synonymes (non éponymiques) se renforcera dans des domaines comme la médecine. Lorsque ces synonymes sont des termes-syntagmes suffisamment longs pour poser des problèmes de transportabilité et de mise en discours, les éponymes serviront de clés d'accès mnémotechniques, un peu à la manière des sigles (cf. «pickwickian syndrome» pour «pulmonary alveolar hypoventilation associated with corpulence» en anglais). Ce cas de figure restera toutefois marginal et il faudrait plutôt envisager le développement à terme d'un système à deux vitesses où les synonymes non éponymiques seront les termes employés par la vaste majorité des scientifiques et le termes éponymiques seront «réservés» en quelque sorte aux grands initiés, aux scientifiques accomplis qui marqueront par là leur droit de «tutoyer» les grands dont les découvertes ont façonné la science.

On peut aussi imaginer que le choix entre éponymes et synonymes non éponymiques soit régi dans certains cas par le contexte du discours. $\grave{A}$ ce propos, on distinguera deux types de contextes: les contextes d'échanges entre scientifiques impliquant une nette négociation du pouvoir («Je suis plus grand savant que vous!») et les contextes d'échanges informatifs neutres. Il nous semble que l'éponyme aura une grande place dans la rhétorique du discours du premier et, tout comme le recours à l'étymologie dans les reformulations, il permettra au scientifique de signifier à son contradicteur que sa connaissance des faits scientifiques est aussi historique, donc plus profonde. Si l'on se permet de revenir un instant sur l'histoire de la découverte des rayons $X$ déjà abondamment évoquée ici, on peut se demander: que reste-t-il de cette reconnaissance de Röntgen avec les éponymes? Combien de fois est-ce qu'on entend en anglais «roentgen rays» à la place de «X-rays»? Il faut le reconnaitre: très peu. À titre d'indice, des 13 éponymes 
reprenant le patronyme de Röntgen dans le Academic Press Dictionary of Science and Technology, seuls quatre sont définis sous les vedettes éponymiques, les autres comportant simplement des renvois à leurs synonymes non éponymiques. Cependant, ces termes éponymiques restent là en réserve et on peut penser que dire «roentgen rays» à la place de «X-rays», c'est d'une certaine façon montrer qu'on connaît l'histoire de la découverte de Röntgen et, comme dans la plupart des cultures, de telles connaissances confèrent à celui qui les détient certains pouvoirs d'intimidation vis-à-vis de ses pairs.

Notes

1. Voir à ce propos la définition que donnait Rey (1979) de la terminologie ou Guilbert (1973) qui rapproche le terme du nom propre.

2. Nos excmples seront tirés indifféremment de l'anglais et du français.

3. Pour des exemples d'éponymes médicaux posant ces problèmes, voir van Hoof (1986).

4. Cet ordre, selon toute vraisemblance, a été dicté par des considérations d'euphonie. Cependant, dans le souci de rétablir la vérité, certains utilisateurs avertis disent aussi «syndrome d'Adams-Stokes», ce qui ne manque pas de créer des problèmes pour l'apprenant.

5. Voir la note 8 pour une explication du couplage fait entre la physique et les mathématiques dans cet article.

6. Dirckx (1983: 79) souligne bien cette importance des éponymes dans la terminologie médicale: «Except for Linnaean taxonomy and perhaps geography, no system of nomenclature ever devised contains as many names of things derived from names of persons as does the language of medicine.»

7. Nous notons par ailleurs qu'on dit aussi «maladie cutanée de Recklinghausen», terme qui semble déplacer le centre d'intérêt de la nature réelle de la maladie vers l'une de ses manifestations, à savoir la présence de tumeurs cutanées. Le terme anglais, «Recklinghausen's disease», qui élimine l'adjectif «nerveux», laisse encore davantage l'utilisateur de la langue face à ses propres moyens.

8. Notre couplage du domaine de la physique avec celui des mathématiques ici et le partage des notions entre les deux qui en résulte reflètent le rapport très étroit entre les deux domaines. On sait que certaines théories de la physique modeme, notamment la mécanique quantique, n'ont d'expression fidèle et complète que mathématique, ce qui explique l'impossibilité de leur vulgarisation (Omnès 1995:50).

9. Il faut souligner ici que la possibilité de systématisation et de structuration en question repose sur les liens étymologiques entre les termes et les formants savants avec lesquels ils sont bâtis, donc sur une motivation strictement en langue. Car les utilisateurs de la langue s'attendent souvent à une certaine communauté de forme entre les termes désignant des concepts proches.

10. L'une des choses qui frappent dans Firkin \& Whitworth (1987) est la disparité dans le traitement des entrées, défaut que les auteurs reconnaissent volontiers et avouent être en partie dû à leurs préférences personnelles: alors que certaines entrées comportent des notes bibliographies s'étendant sur plusieurs pages, d'autres n'en ont pas du tout ou n'ont droit qu'à cinq lignes.

11. Le temps pyschologique est entendu ici au sens de l'appréciation par le sujet de l'adéquation entre une tâche et le temps passé à l'accomplir.

12. Même le titre choisi par Hamburger (1982) pour le chapitre de son ouvrage consacré aux éponymes, «Éponymanie», en dit long sur cette attitude vis-à-vis de l'éponyme.

13. Ce point nous paraît suffisamment important pour être souligné ici. Car c'est parce qu'il y a derrière tout éponyme un état civil et un curriculum vite qu'il y a très peu de risques de confusion pour savoir quels sont les scientifiques qui sont honorés dans «Agassiz Lake» (terme de géologie) et «Agassiz trawl» (terme d'océanographie). Le premier, terme désignant le lac pléistocène qui occupait l'emplacement du bassin de l'actuel River Red de Mantinoba et ses environs, ne peut être qu'en hommage à Louis Agassiz (18071873), le naturaliste et géologue, et le second, terme désignant un appareil d'échantillonnage en milieu marin mis au point par un Agassiz pour l'expédition sur Blake en 1877, en hommage à Alexander Agassiz (1835-1907), spécialiste de zoologie marine et ingénieur des mines.

14. Il y a certes la possibilité du recours à la création ex-nihilo, mais il s'agit d'une option trop utopique.

15. Voir aussi «maladie de Hansen» et «syndrome de Down», dont le maintien s'expliquerait par le fait qu'ils sont des synonymes plus politiquement corrects que «lèpre» et «mongolisme» (Dirckx 1983).

16. «Scientific recognition takes a variety of forms, graded to the various stages of a successful career. At the very lowest level, an academic scientist scarcely exists unless his or her work has been published in a reputable scientific journal. But a published paper is of little significance unless it is cited in papers by other scientists [...] The next level is more subtle, being signalled by the attribution of some phenomenon or concept to a particular researcher, indicating that it is likely to prove a permanent item of established knowledge [...] This is often recognized eponymously, by attaching to it the name of the discoverer, as in Addison's disease, Bankasia, Einstein's equation, etc. [...]» (Ziman 1984: 70)

17. Notons au passage que l'exégèse de Dirckx (1983) n'est pas en accord avec celle de Hamburger: «The pickwickian syndrome takes its name from Charles Dickens's Pickwick Papers. The reference is not to the 
title character but to Mr. Wardle's servant Joe, usually called simply 'the fat boy,' who falls asleep whenever he stops moving, even when he is on his feet. The connection between Mr. Pickwick and the disorder that bears his name (pulmonary alveolar hypoventilation associated with corpulence) is thus exceedingly tenuous.» (Dirckx 1983: 74)

18. On est là dans un cas limite du phénomène puisque, étymologiquement, on ne devrait parler d'éponyme que Iorsqu'il y a emprunt d'un patronyme.

19. Ziman (1984: 59) définit par ailleurs la communication scientifique primaire en ces termes: «A primary scientific communication is an original contribution to knowledge, by a named author or authors, normally published as a paper or article, of limited length [...] in a periodical, or journal devoted to a specific subject.»

20. Guinier (1975: 87) explique l'attitude du public vis-à-vis de la découverte de Röntgen en ces termes: «[...] Röntgen a ressuscité, mutatis mutandis, le mythe de Prométhée. La nature - Dieu, selon d'aucuns - a fixé une limite à notre connaissance du monde, puisque la lumière à laquelle notre oeil est sensible est arrêtée à la surface d'à peu près toute matière. Et voilà qu'un homme force cet interdit, dévoile ce qui est caché dans une boîte de bois, et révèle l'intérieur de notre corps.»

21. Déjà en février 1896, la très respectable revue JAMA parlait de «Roentgen's photography». Il est certes difficile aujourd'hui d'établir la datation exacte des différents éponymes relatifs à la technique des rayons $\mathrm{X}$ empruntant le nom de Röntgen, mais on en dénombre la bagatelle de 13 dans le Academic Press Dictionary of Science and Technology, plus «roentgencinematography» qui n'est pas listé par le dictionnaire.

22. Parce que les termes éponymiques manquent de la motivation en langue qu'on constate dans les termes issus de la composition savante, il est beaucoup plus difficile de se rendre compte du moment où le contenu du concept désigné par le terme change. Dirckx (1983: 84) écrit à ce propos: «An eponym is rather more liable to undergo semantic mutation than a term coined from meaningful language material.»

23. On rappelle à l'instar d'Alford (1995) que Whorf était physicien et était bien au courant de la relativité d'Einstein, qui a influencé son étude sur les langues et cultures de certaines tribus amérindiennes, surtout les Hopi.

24. Firkin \& Whitworth $(1987: v)$ racontent une anecdote fort intéressante concernant le «syndrome de Guillain-Barré». En apprenant que le syndrome était désigné dans certains lieux comme «syndrome de Landry-Guillain-Barré», Guillain aurait observé : «c'est une confusion nosographique». Or, la communication originelle où le syndrome fut décrit pour la première fois, «Sur un syndrome de radiculo-neurite avec hyperalbumose du liquide céphalo-rachidien sans réaction cellulaire. Remarques sur les caractères cliniques et graphiques des réflexes tendinaux.» (Buil. Soc. Med. Hop. Paris, 14: 1462-1470), avait été signée par trois auteurs: Guillain, Barré et Stohl. Cependant, dans le partage du «magot» («syndrome de Guillain-Barré»), le troisième co-auteur avait été oublié.

25. On a pu en avoir un aperçu mais sur un autre plan avec la récente controverse franco-américaine portant sur la paternité de la découverte du virus responsable du SIDA, le HIV.

26. À propos de la découverte des rayons $X$, on pourrait se demander si parier de «roentgen rays» et de «roentgenogram», ce n'est pas oublier vite que la découverte de Röntgen en tant que telle était pratiquement inutilisable et hors de portée (en 1896, il fallait entre 20 et 60 minutes pour faire une seule radiographie, l'appareil coûtait plus de 100000 dollars et le niveau d'irradiation à laquelle étaient exposés les sujets et les radiologues était tel qu'il fallait vraiment que l'urgence de l'examen mérite les risques encourus) et que c'est grâce aux efforts sans relâche d'autres scientifiques que la technique est ce qu'elle est aujourd'hui.

27. N'est-ce pas le père de la relativité lui-même qui se lamentait : «Pour me punir pour mon manque d'égard pour l'autorité, Dieu m'en a fait une»?

\section{RÉFÉRENCES}

ALFORD, Dan (1995): «Disc: Sapir-Whorf and what to tell students these days», Linguist, Vol-6-1149, Aug 22 1995, Internet http: // www.ling.edu / linguist / 6-1149.

BALLENTYNE, D. W. G. \& D. R. LOVETT (1970): A Dictionary of Named Effects and Laws, 3rd Edition, London, Chapman and Hall.

BLACKWELDER, Richard E. (1967): Taxonomy. A Text and Reference Book, New York, John Wiley.

BYNUM, W. F., BROWNE, E. J. \& Roy PORTER (Eds.) (1983): Macmillan Dictionary of the History of Science, London, Macmillan.

CHUKWU, Uzoma (1993): Le Repérage des termes dans un corpus bilingue anglais / français, Thèse de Doctorat Nouveau Régime, Faculté des Langues, Université Lumière - Lyon 2.

COTTEZ, Henri (1994): «Les bases épistémologiques et linguistiques de la nomenclature chimique de 1787», Meta, 39 (4), pp. 676-691

DIRCKX, John H. (1983): The Language of Medicine. Its Evolution, Structure, and Dynamics, 2nd Edition, New York, Praeger. 
FIRKIN, B. G. \& J. A. WHITWORTH (1987): Dictionary of Medical Eponyms, Camforth (U.K.), The Parthenon Publishing Group.

GUILBERT, Louis (1973): «La spécificité du terme scientifique et technique», Langue française, 17, 5.18.

GUINIER, André (1995): «Les étranges rayons du Professeur Röntgen», La Recherche, 281 (novembre 1995), pp. 86-89.

HAMBURGER, Jean (1982): Introduction au langage de la médecine, Paris, Flammarion, coll. «MédecineSciences».

MORRIS, Christopher (1992): Academic Press Dictionary of Science and Technology, San Diego, Academic Press.

OMNÈS, Roland (1995): «Une nouvelle interprétation de la mécanique quantique. Retrouver la physique classique sans rien admettre d'autre que les lois quantiques», La Recherche, 280 (octobre 1995), pp. 50-56.

PAJAUD, Daniel (1989): La taxinomie bionaturaliste. 1. Nomenclature et taxinomie. Pratiques de la dénomination et usages des concepts en ontologie, Paris, Gauthier.

REY, Alain (1979): La terminologie : noms et notions, coll. «Que sais-je?» $\mathrm{n}^{\circ} 1780$, Paris, PUF, $127 \mathrm{p}$.

STERPELLONE, Luciano (1986): Instruments for Health. From Origins to Yesterday, Milan, Farmitalia Carlo Erba.

VAN HOOF, Henri (1986): «Les éponymes médicaux : essai de classification», Meta, 31 (1), pp. 59-84.

ZIMAN, John (1984): An Introduction to Science Studies, London, C.U.P. 